\title{
How to get the weak order out of a digraph?
}

\author{
Francois Viard $\mathbb{1}^{\dagger}$ \\ ${ }^{1}$ Université Lyon 1, Institut Camille Jordan, UMR 5208 du CNRS, 69622 Villeurbanne, France
}

\begin{abstract}
We construct a poset from a simple acyclic digraph together with a valuation on its vertices, and we compute the values of its Möbius function. We show that the weak order on Coxeter groups $A_{n-1}, B_{n}, \widetilde{A}_{n}$, and the flag weak order on the wreath product $\mathbb{Z}_{r}\left\{S_{n}\right.$ introduced by Adin, Brenti and Roichman (2012), are special instances of our construction. We conclude by briefly explaining how to use our work to define quasi-symmetric functions, with a special emphasis on the $A_{n-1}$ case, in which case we obtain the classical Stanley symmetric function.

Résumé. On construit une famille d'ensembles ordonnés à partir d'un graphe orienté, simple et acyclique munit d'une valuation sur ses sommets, puis on calcule les valeurs de leur fonction de Möbius respective. On montre que l'ordre faible sur les groupes de Coxeter $A_{n-1}, B_{n}, \widetilde{A}_{n}$, ainsi qu'une variante de l'ordre faible sur les produits en couronne $\mathbb{Z}_{r} 2 S_{n}$ introduit par Adin, Brenti et Roichman (2012), sont des cas particuliers de cette construction. On conclura en expliquant brièvement comment ce travail peut-être utilisé pour définir des fonction quasi-symétriques, en insistant sur l'exemple de l'ordre faible sur $A_{n-1}$, où l'on obtient les séries de Stanley classiques.
\end{abstract}

Keywords: Tableaux, digraphs, posets, Coxeter groups, weak order, reduced decompositions.

\section{Introduction}

A tableau is a map from a finite subset $S \subset \mathbb{N} \times \mathbb{N}$ to $\mathbb{N}$. Some families of tableaux are very classical in combinatorics and algebra, such as standard and semi-standard tableaux which play a fundamental role in the study of symmetric functions and representation theory.

In [4], Edelman and Greene introduced a new family of tableaux called balanced and exhibited a beautiful connection between them and the theory of reduced decompositions in the symmetric groups. This study has been extended by Fomin et al. in [5] by considering balanced labellings of the Rothe diagram of a permutation. Those labelled diagrams are a convenient way to visualise both the inversion set of a permutation and its associated set of reduced decompositions, and can be interpreted as a generalization of standard Young tableaux. Moreover, they introduced column-strict balanced labellings which generalize the notion of semi-standard Young tableaux. Column-strict labellings of the diagram of a permutation $\sigma$ yield the Stanley symmetric function $F_{\sigma}$ (introduced in [7]) in the same way semi-standard tableaux yield Schur functions. This approach has been generalized to the affine Coxeter group $\widetilde{A}_{n}$ by Yun and Yoo in [10], and leads to a combinatorial description in terms of tableaux of the affine Stanley symmetric function introduced by Lam in [6].

\footnotetext{
${ }^{\dagger}$ Email: Viardemath. univ-lyonl. fr. 
The main purpose of this paper is to introduce a new family of posets, defined from a simple acyclic digraph together with a valuation on its vertices, by using an iterative process which peels the graph vertex by vertex. Then, we exhibit some general properties of these posets: we show that they are complete meet semi-lattice, and we give an explicit formula to compute the values of their Möbius function. Then we give some examples of classical posets which are special instances of this construction, namely the weak order on $A_{n-1}, B_{n}, \widetilde{A}_{n}$, the flag weak order on $\mathbb{Z}_{r} 2 S_{n}$ introduced by Adin, Brenti and Roichman (2012, [1]), the tree order of any tree, and the up-set (resp. down-set) lattice of any finite poset. In the $A_{n-1}$ case we explain how this description naturally leads to associate a family of tableaux to each element of $A_{n-1}$, and we explain how they can be used to find the result of [5] again. To finish we mention that a similar method can be applied to the $\widetilde{A}_{n}$ case, and we briefly mention how these combinatorial consequences can be generalized to the other examples.

\section{Definition of the family of posets}

We begin with some standard definitions about poset theory and graph theory. A poset is a couple $\mathcal{P}=$ $(P, \leq)$, where $P$ is a set and $\leq$ is a binary relation which is reflexive, antisymmetric, and transitive. A poset $\mathcal{P}$ is called a complete meet (resp. join) semi-lattice if and only if every subset $S$ of $\mathcal{P}$ has an infimum (resp. supremum) in $\mathcal{P}$, i.e. there exists $z$ in $\mathcal{P}$ such that if $y \in \mathcal{P}$ and $y \leq x$ (resp. $x \leq y$ ) for all $x \in S$, then $y \leq z$ (resp. $z \leq y$ ). If $\mathcal{P}$ is both a complete join and meet semi-lattice, then we say that $\mathcal{P}$ is a complete lattice. A lower set of $\mathcal{P}$ is a subset $A$ of $\mathcal{P}$ such that for all $x \in A$ and $y$ in $P$, if $y \leq x$ then $y \in A$. It is classical that the lower sets of $\mathcal{P}$ ordered by inclusion is a lattice (for more details about poset theory, see [8]).

A simple digraph is a couple $G=(V, E)$, where $V$ is the set of vertices of $G$, and $E$ is a subset of $V \times V$ called the set of arcs of $G$. A cycle of $G$ is a finite sequence $x_{1}, \ldots, x_{n}$ of vertices of $G$ such that $\left(x_{i}, x_{i+1}\right) \in E$ for all $i \in \mathbb{N}$, where the indices are taken modulo $n$. A graph $G$ is called acyclic if it does not have any cycle. On each vertex $x$ of $G$, we define the statistic

$$
d^{+}(x)=|\{y \mid(x, y) \in E\}|
$$

called the out-degree of $x$, and which is possibly infinite.

\subsection{General idea of the construction}

We first present a suitable method to construct all lower sets of a finite poset $\mathcal{P}=(P, \leq)$, using the notion of linear extension of $\mathcal{P}$. A linear extension of $\mathcal{P}$ is an injective sequence $\left[a_{1}, \ldots, a_{n}\right]$ (with $|P|=n$ ) of elements of $P$ such that: if $a_{i} \leq a_{j}$ in $\mathcal{P}$, then $i \leq j$. It is classical that $A \subseteq P$ is a lower set of $\mathcal{P}$ if and only if there exists $\left[a_{1}, \ldots, a_{n}\right]$ a linear extension of $\mathcal{P}$ and $1 \leq k \leq n$ such that $A=\left\{a_{1}, \ldots, a_{k}\right\}$ (see [8]). Then to construct all the lower sets of $\mathcal{P}$, we just have to construct all the linear extensions of $\mathcal{P}$, and this can easily be done recursively thanks to the following iterative method.

Since $\mathcal{P}$ is finite, there exists $a_{1}$ in $\mathcal{P}$ which is a minimum, that is if $x \leq a_{1}$ in $\mathcal{P}$, then $x=a_{1}$. Set $\mathcal{P}_{2}=\left(P \backslash\left\{a_{1}\right\}, \leq\right)$ the finite poset obtained by suppressing $a_{1}$ in $\mathcal{P}$. Then there exists $a_{2}$ in $\mathcal{P}_{2}$ which is a minimum. Then we can consider the poset $\mathcal{P}_{3}$ obtained by suppressing $a_{2}$ in $\mathcal{P}_{2}$, and so on. At the end we obtain an injective sequence $\left[a_{1}, \ldots, a_{n}\right]$ of elements of $\mathcal{P}$. By construction, this sequence is a linear extension of $\mathcal{P}$. Furthermore, one can easily prove by induction that all the linear extensions of $\mathcal{P}$ can be obtained by this way. 
In a certain sense, this method "peels" a finite poset element by element, in order to obtain a family of sequences which give rise to an interesting family of sets (here, the lower sets). What we propose here it is to apply a similar principle to a simple acyclic digraph. Namely, we will peel the digraph vertex by vertex, with respect to a constraint given by a valuation on its vertices. It will give rise to a family of sequences of vertices of the graph, then to a family of subsets having an interesting poset structure once ordered by inclusion.

\subsection{Construction of the family of posets}

We start by defining the valuation on the vertices of a simple acyclic digraph.

Definition 2.1 Set $G=(V, E)$ a simple acyclic digraph. A valuation $\theta: V \rightarrow \mathbb{N}$ is called an out-degree compatible valuation of $G$ (abbreviated in " $O C V$ ") if and only if for all $x \in V$, we have $0 \leq \theta_{x} \leq d^{+}(x)$.

In what follows, we will denote $\mathcal{G}=(G, \theta)$ the pair of a simple acyclic digraph $G$ together with an OCV of $G$. Thanks to the associated OCV, some vertices of $G$ will be of special interest.

Definition 2.2 (Erasable vertex) A vertex $x$ of $G$ is called erasable in $\mathcal{G}$ if and only if:

- $\theta_{x}=0$;

- for all $z \in V$ such that $(z, x) \in E$, we have $\theta_{z} \neq 0$.

Now we define the peeling sequences of $\mathcal{G}$, which leads to the definition of the claimed poset.

Definition 2.3 (Peeling process and peeling sequences) We construct recursively two sequences: a sequence $L=\left[x_{1}, x_{2}, \ldots\right]$ of elements of $V$, and a sequence $\left(\mathcal{G}_{i}=\left(G_{i}, \theta^{i}\right)\right)_{1 \leq i \leq n}$ as follows.

1. $\operatorname{Set} \mathcal{G}_{1}=\mathcal{G}$.

2. If there is not any erasable vertex in $\mathcal{G}_{i}$, the process stop. Otherwise, choose $x$ a vertex of $G_{i}$ which is erasable in $\mathcal{G}_{i}$, and set $x_{i}=x$.

(a) Set $G_{i+1}$ the simple acyclic directed graph obtained by suppressing the vertex $x_{i}$ in $G_{i}$ together with all the arcs of $G_{i}$ containing it.

(b) Set $\theta^{i+1}$ the OCV of $G_{i+1}$ such that $\theta_{y}^{i+1}=\theta_{y}^{i}-1$ if $(y, x)$ is an arc of $G_{i}$, and $\theta_{y}^{i+1}=\theta_{y}^{i}$ otherwise. Then set $\mathcal{G}_{i+1}=\left(G_{i+1}, \theta^{i+1}\right)$ and go back to the Step 2.

A sequence $L$ which comes from this process is called a peeling sequence of $\mathcal{G}$, and we denote $P S(\mathcal{G})$ the set of all the peeling sequences of $\mathcal{G}$.

And now we give the definition of the poset.

Definition 2.4 Let $L=\left[x_{1}, x_{2}, \ldots\right]$ be a peeling sequence of $\mathcal{G}$. The initial sections of $L$ are the sets of the form $\left\{x_{1}, x_{2} \ldots, x_{k}\right\}$ for some $k \in \mathbb{N}^{*}$. By convention, $\emptyset$ is an initial section of $L$. We set $I S(\mathcal{G})$ the set of all the initial sections of all the peeling sequences of $\mathcal{G}$, and we denote $\mathcal{P}(\mathcal{G})=(I S(\mathcal{G}), \subseteq)$ the poset obtained by ordering $I S(\mathcal{G})$ by inclusion.

Example 2.2.1 Consider $\mathcal{G}$ as depicted in the upper left corner of the following figure. The peeling sequences of $\mathcal{G}$ are $L_{1}=[a, c, b]$ and $L_{2}=[b, c, a]$, thus $I S(\mathcal{G})=\{\emptyset,\{a\},\{b\},\{a, c\},\{b, c\},\{a, b, c\}\}$. 

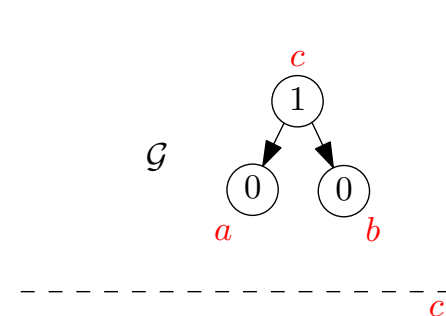

Sequence $\left(\mathcal{G}_{i}\right)$

associated with $L_{1}$.
0

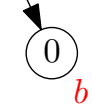

$\mathcal{G}_{2}$

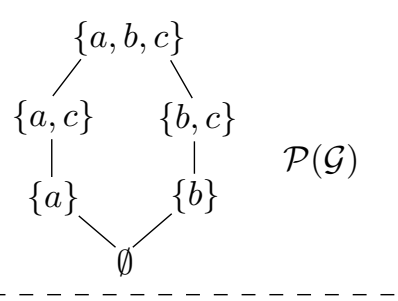

$\emptyset$

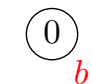

$\mathcal{G}_{3}$

$\mathcal{G}_{4}$

\section{General properties of $\mathcal{P}(\mathcal{G})$}

In this section we give the main properties of the poset $\mathcal{P}(\mathcal{G})$. We start by announcing our results, and the scheme of the proofs will be given in the sequel.

Theorem 3.1 For all $\mathcal{G}=(G, \theta)$, the poset $\mathcal{P}(\mathcal{G})$ is a graded complete meet semi-lattice, and its rank function is $\rho: A \rightarrow|A|$. Moreover, when the underlying graph $G$ is finite, $\mathcal{P}(\mathcal{G})$ is a complete lattice.

In the context of these posets, it is possible to give an explicit formula for the values of their Möbius function. Recall that the Möbius function on a graded poset $\mathcal{P}=(P, \leq)$ is the application $\mu: P^{2} \rightarrow \mathbb{N}$ recursively defined by $\mu(x, x)=1$ for all $x \in P$, and $\sum_{x \leq z \leq y} \mu(x, z)=0$ for all $(x, y) \in P^{2}$.

For the sake of clarity, we give the formula only for the couples of the form $(\emptyset, A), A \in I S(\mathcal{G})$, but a similar one can be stated for all couples in $I S(\mathcal{G})$.

Theorem 3.2 Set $A \in I S(\mathcal{G})$. We denote $\mathcal{N}(A)=\left\{x \in A \mid \theta_{x}=0\right\}$, and $\mathcal{F}(A)=\{x \in A \mid A \backslash\{x\} \in$ $I S(\mathcal{G})\}$. Then we have the two following possibilities:

1. if $\mathcal{F}(A)=\mathcal{N}(A)$, then $\mu(\emptyset, A)=(-1)^{|\mathcal{N}(A)|}$;

2. otherwise, $\mu(\emptyset, A)=0$.

\subsection{Proof of Theorem 3.1}

The proof of this theorem has to be divided into several steps. The first one is a characterization of the elements of $I S(\mathcal{G})$.

Proposition 3.1.1 Set $A$ a finite subset of vertices of $G$. We have that $A \in I S(\mathcal{G})$ if and only if the two following conditions are satisfied:

(1) for all $x \in A, \theta_{x} \leq \mid\{y \mid y \in A$ and $(x, y) \in E\} \mid$;

(2) for all $x \in V \backslash A, \theta_{x} \geq \mid\{y \mid y \in A$ and $(x, y) \in E\} \mid$. 
Proof: The proof of the necessary condition consists in a straightforward verification using the construction of a peeling sequence. The reverse direction is proved by induction on $|A|$ : essentially, the fact that $G$ is acyclic together with condition (1) and (2) imply that there exists $x$ in $A$ which is erasable in $\mathcal{G}$. Then we begin the peeling process by picking this vertex and we set $\mathcal{G}^{\prime}$ the obtained digraph and OCV. One can show that $A \backslash\{x\}$ satisfies conditions (1) and (2) in $\mathcal{G}^{\prime}$, and the induction hypothesis applies. Hence $A \backslash\{x\} \in I S\left(\mathcal{G}^{\prime}\right)$ and this concludes the proof.

The next proposition implies immediately that $\mathcal{P}(\mathcal{G})$ is a graded poset with rank function $\rho: A \mapsto|A|$.

Proposition 3.1.2 Set $A$ and $B$ two elements of $I S(\mathcal{G})$, and denote $k=|A|$ and $q=|B|$. If $A \subseteq B$, then there exists $L=\left[x_{1}, x_{2}, \ldots\right] \in P S(\mathcal{G})$ such that $A=\left\{x_{1}, \ldots, x_{k}\right\}$ and $B=\left\{x_{1}, \ldots, x_{q}\right\}$.

Proof: We suppose that $k<q$. We construct explicitly the sequence $L$ as follows. Since $A \in I S(\mathcal{G})$, we can start by picking the first $k$ elements $x_{1}, \ldots, x_{k}$ in $A$. Set $\mathcal{G}^{\prime}=\left(G^{\prime}, \theta^{\prime}\right)$ obtained from $\mathcal{G}$ after this procedure. Thus $B \backslash A$ is a finite subset of vertices of $G^{\prime}$, moreover $B \backslash A$ satisfy the conditions of Proposition 3.1.1. hence $B \backslash A \in I S\left(\mathcal{G}^{\prime}\right)$. So there exists $L^{\prime}=\left[x_{k+1}, \ldots, x_{q}, \ldots\right] \in P S\left(\mathcal{G}^{\prime}\right)$ such that $B \backslash A=\left\{x_{k+1}, \ldots, x_{q}\right\}$. At the end if we concatenate the sequences $\left[x_{1}, \ldots, x_{k}\right]$ and $L^{\prime}$, we obtain $L=\left[x_{1}, x_{2}, \ldots\right]$ which is a peeling sequence of $\mathcal{G}$ by construction, and satisfies all the claimed properties.

We conclude by an explicit construction of the infimum (also called the meet) of a set $S \subseteq I S(\mathcal{G})$. We start by a useful lemma, which is an immediate consequence of Proposition 3.1.1.

Lemma 3.1.1 Set $S \subseteq I S(\mathcal{G})$, and denote $X:=\bigcap_{A \in S} A$. If there exists $x \in X$ such that $\theta_{x}=0$, then there exists $z \in X$ which is erasable in $\mathcal{G}$.

Construction of the meet. We consider $S$ and $X$ as defined in the previous lemma, and we construct a set $C \in I S(\mathcal{G})$ as follows. If for all $x \in X, \theta_{x} \neq 0$, we set $C=\emptyset$. Otherwise, let $z_{1} \in X$ be an erasable vertex of $\mathcal{G}$. We start the peeling process by picking this vertex and we denote $\mathcal{G}_{2}=\left(G_{2}, \theta^{2}\right)$ the obtained graph and OCV. Then for all $A \in S, A \backslash\left\{z_{1}\right\} \in I S\left(\mathcal{G}_{2}\right)$, so we can again apply Lemma 3.1.1 to $X \backslash\left\{z_{1}\right\}$ seen as a subset of vertices of $G_{2}$. If for all $x \in X \backslash\left\{z_{1}\right\}, \theta_{x}^{2} \neq 0$ we set $C=\left\{z_{1}\right\}$; otherwise, let $z_{2} \in X \backslash\left\{z_{1}\right\}$ be an erasable vertex of $\mathcal{G}_{2}$ and we perform the peeling process by picking this vertex. We repeat this procedure until there is not any erasable vertex left (this process always ends, since $X$ is finite), and we set $C$ the obtained set. By construction, $C \in I S(\mathcal{G})$.

At first glance, this set $C$ seems to depend heavily on the choices of vertices made at each step of its construction. The next proposition shows that it is not the case.

Proposition 3.1.3 Set $U \in I S(\mathcal{G})$. If $U \subseteq X$, then $U \subseteq C$.

Proof: Since $U \in I S(\mathcal{G})$, there exists $L=\left[x_{1}, x_{2}, \ldots\right] \in P S(\mathcal{G})$ and $k \in \mathbb{N}^{*}$ such that $U=$ $\left\{x_{1}, \ldots, x_{k}\right\}$. Assume that $U \not \subset C$ and set $j \geq 1$ minimal such that $x_{j} \notin C$. Then by construction of $C$ and Lemma 3.1.1, $x_{j}$ has to be in $C$, which is a contradiction.

Since $C \subseteq X$ by construction, the previous proposition leads to the following corollary.

Corollary 3.1.1 The set $C$ is the infimum of $S$. 
When the underlying graph $G$ is finite, we obviously have that $V$, the set of all the vertices of $G$, satisfies the conditions of Proposition 3.1.1, hence $V \in I S(\mathcal{G})$, and the poset $\mathcal{P}(\mathcal{G})$ is bounded. Since it is a meet semi-lattice, then $\mathcal{P}(\mathcal{G})$ is a lattice, and this concludes the proof of Theorem 3.1

\subsection{Proof of Theorem 3.2}

The proof of this formula is purely combinatorial, and is based on the well-known inclusion-exclusion principle (see [8]). For all $B \in I S(\mathcal{G})$, we denote $[\emptyset, B]=\{U \in I S(\mathcal{G}) \mid \emptyset \subseteq U \subseteq B\}$. Set $A, \mathcal{N}(A)$ and $\mathcal{F}(A)$ as defined in Theorem 3.2 For all $S \subseteq \mathcal{F}(A)$, we denote $A_{S}$ the infimum of $\{A \backslash\{x\} \mid x \in$ $S\} \subseteq I S(\mathcal{G})$. We have the following lemma.

Lemma 3.2.1 Set $S \subseteq \mathcal{F}(A)$. We have that $A_{S} \neq \emptyset$ if and only if $\mathcal{N}(A) \not \subset S$.

Proof: We have that the intersection of all the $A \backslash\{x\}$, for $x$ in $S$, is equal to $A \backslash S$. By Lemma 3.1.1, if $\mathcal{N}(A) \not \subset S$ then there exists $z \in A \backslash S$ which is erasable in $\mathcal{G}$, so the infimum of $\{A \backslash\{x\} \mid x \in S\}$ is not $\emptyset$. The reciprocal is based on similar arguments.

An immediate consequence of the meet semi-lattice structure of $\mathcal{P}(\mathcal{G})$ is that, for all $U$ and $V$ in $I S(\mathcal{G})$, $[\emptyset, U] \cap[\emptyset, V]=[\emptyset, U \wedge V]$ where $U \wedge V$ is the infimum of $\{U, V\}$. This basic remark leads to the claimed formula: first, we obviously have

$$
[\emptyset, A] \backslash\{A\}=\bigcup_{x \in \mathcal{F}(A)}\left[\emptyset, A_{\{x\}}\right]
$$

Then, by the inclusion-exclusion principle we have that

$$
|[\emptyset, A] \backslash\{A\}|=\sum_{\emptyset \neq S \subseteq \mathcal{F}(A)}(-1)^{|S|+1}\left|\bigcap_{x \in S}\left[\emptyset, A_{\{x\}}\right]\right|=\sum_{\emptyset \neq S \subseteq \mathcal{F}(A)}(-1)^{|S|+1}\left|\left[\emptyset, A_{S}\right]\right| .
$$

Once applied to the Möbius function of $\mathcal{P}(\mathcal{G})$, this gives rise to the following identity:

$$
\mu(\emptyset, A)=-\sum_{\emptyset \neq S \subseteq \mathcal{F}(A)}(-1)^{|S|+1} \sum_{B \in\left[\emptyset, A_{S}\right]} \mu(\emptyset, B) .
$$

By definition of the Möbius function, $\sum_{B \in\left[\emptyset, A_{S}\right]} \mu(\emptyset, B)=1$ if $A_{S}=\emptyset$, and 0 otherwise. Hence by Lemma 3.2.1. if $\mathcal{N}(A) \not \subset \mathcal{F}(A)$, then $\mu(\emptyset, A)=0$. As a consequence, Equation (11) becomes

$$
\begin{gathered}
\mu(\emptyset, A)=-\sum_{\mathcal{N}(A) \subseteq S \subseteq \mathcal{F}(A)}(-1)^{|S|+1}=(-1)^{|\mathcal{N}(A)|} \sum_{S \subseteq \mathcal{F}(A) \backslash \mathcal{N}(A)}(-1)^{|S|} \\
=(-1)^{|\mathcal{N}(A)|}(1-1)^{|\mathcal{F}(A) \backslash \mathcal{N}(A)|} .
\end{gathered}
$$

The claimed formula follows immediately. 


\section{Applications of this construction}

Let $W$ be a Coxeter group with finite generating set $S$, and Coxeter matrix $M=\left(m_{s t}\right)_{s, t \in S}$. That is, $M$ is a symmetric matrix with $m_{s s}=1$ and, for $s \neq t, m_{s t}=m_{t s} \in\{2,3, \ldots\} \cup\{\infty\}$. The relations among the generators are of the form $(s t)^{m_{s t}}=1$ if $m_{s t}<\infty$. For $w \in W$ we denote by $\ell(w)$ the minimum length of any decomposition $w=s_{1} \cdots s_{l}$ with $s_{i} \in S$. Such decompositions with minimum length are called reduced, and we denote by $\mathcal{R}(w)$ the set of all reduced decompositions of $w$.

This statistic $\ell$ on $W$ is the rank function of a poset structure $\left(W, \leq_{R}\right)$ called the (right) weak order on $W$, and which is defines as follows: we say that $w \leq_{R} \tau$ if and only if there exists $s_{1}, \ldots, s_{k}$ in $S$ such that $\tau=\omega s_{1} \cdots s_{k}$ and $\ell(\tau)=\ell(w)+k$. It is classical that $\left(W, \leq_{R}\right)$ is a complete meet semi-lattice when $W$ is infinite, a complete lattice when $W$ is finite, and that its Möbius function take values into $\{ \pm 1,0\}$ (see [2] and [3]). Hence, it is natural to look for an interpretation of the weak order through the theory developed in the previous section. Indeed, we can provide such a description in some cases. In the next theorem, we give a list of posets which admit such an interpretation.

Theorem 4.1 For each poset $\mathcal{P}$ in the following list, there is an explicit $\mathcal{G}=(G, \theta)$ such that $\mathcal{P}$ is isomorphic to $\mathcal{P}(\mathcal{G})$.

- The (right) weak order on Coxeter groups $A_{n-1}, B_{n}$ and $\widetilde{A}_{n}$.

- The flag weak order on $\mathbb{Z}_{r}\left\{S_{n}\right.$ (see [1]).

- The up-set (resp. down-set) lattice of any finite poset.

- The tree order of any finite tree.

In this extended abstract we will focus on the case of the symmetric group $A_{n-1}$.

\subsection{The weak order on $A_{n-1}$}

Recall that $A_{n-1}$ is the Coxeter group with generating set $S=\left\{s_{1}, \ldots, s_{n-1}\right\}$, and with Coxeter matrix given by $m_{s_{i} s_{i+1}}=3$ for all $1 \leq i \leq n-2$, and $m_{s t}=2$ otherwise. One can see $A_{n-1}$ as the symmetric group $S_{n}$ by identifying the generator $s_{i}$ to the transposition of $S_{n}$ which exchanges the integers $i$ and $i+1$. The main issue that arises when we want to find a pair $\mathcal{G}=(G, \theta)$ such that $\left(A_{n-1}, \leq_{R}\right)$ is isomorphic to $\mathcal{P}(\mathcal{G})$, is that on the one side we have a poset whose elements are permutation (once $A_{n-1}$ identified to the symmetric group $S_{n}$ ), and on the other side we have a poset whose elements are sets. Fortunately, there is a canonical set associated with each permutation $\sigma \in S_{n}$, its inversion set:

$$
\operatorname{Inv}(\sigma)=\left\{(a, b) \in\{1, \ldots, n\}^{2} \mid a<b \text { and } \sigma^{-1}(a)>\sigma^{-1}(b)\right\} .
$$

There is a deep connection between inversion sets and the weak order on $S_{n}$. That is, if $\sigma$ and $\omega$ are two elements of $S_{n}$, then $\sigma \leq_{R} \omega$ if and only if $\operatorname{Inv}(\sigma) \subseteq \operatorname{Inv}(\omega)$. This property allows us to clarify our goal: we are looking for a pair $\mathcal{G}=(G, \theta)$ such that,

1. the vertices of the graph are indexed by the couple of integers $(a, b) \in\{1, \ldots, n\}^{2}$ such that $a<b$;

2. the digraph structure of $G$, together with the valuation $\theta$, imply that $I S(\mathcal{G})$ is constituted exactly of the sets of the form $\operatorname{Inv}(\sigma), \sigma \in S_{n}$. 
There is a convenient way to represent the set $\left\{(a, b) \in\{1, \ldots, n\}^{2} \mid a<b\right\}$, by considering the $n$-th staircase diagram, namely the Ferrers diagram of the partition $\lambda_{n}=(n-1, n-2, \ldots, 1)$ of size $N=\left(\begin{array}{l}n \\ 2\end{array}\right)$. At the left of the next figure, the diagram associated to the case $n=5$ is represented. The coordinates of each box can be read thanks to the circled integers on the diagonal.

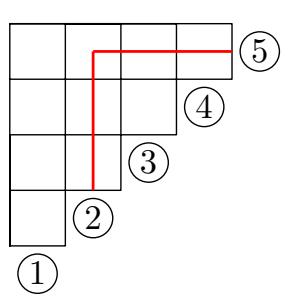

The coordinates of this box are $(2,5)$

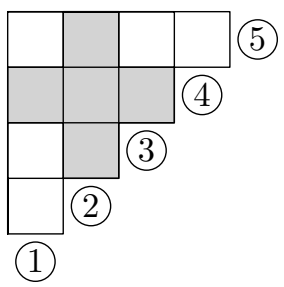

The inversion set of $\sigma=[4,1,3,5,2]$

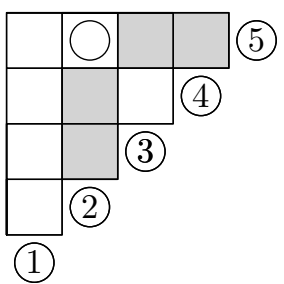

Hook based on $(2,5)$

As depicted in the middle of the figure, one can easily visualize the inversion set of any element of $S_{n}$ as a subset of boxes in $\lambda_{n}$. Note that the set constituted by all the boxes of the diagram corresponds to the inversion set of the reverse permutation $[n, n-1, \ldots, 1] \in S_{n}$, which is the maximal element in the weak order.

Definition 4.1 We define a digraph structure $G$ on this diagram (where the vertices are the boxes of the diagram), thanks to a classical combinatorial object associated to each box $c$, the hook based on $c$, denoted $\mathcal{H}(c)$ and composed of all the boxes which are on the right and below $c$ (represented at the right of the Figure): we say that there is an arc from $c$ to $d$ if and only if $c \neq d$ and $d \in \mathcal{H}(c)$. The obtained digraph $G$ is obviously a simple acyclic digraph whose vertices are the boxes of the diagram. Moreover, with this definition we have that the out-degree of any box is an even number. Hence we set $\theta$ the valuation $\theta: c \mapsto \frac{d^{+}(c)}{2}$. Finally, we denote $\mathcal{G}^{A}=(G, \theta)$.

Among others natural choices for a valuation on this digraph, this one is in fact the "good" choice as stated in the next theorem.

Theorem 4.2 Set $n \in \mathbb{N}$ and $\mathcal{G}^{A}$ associated with $\lambda_{n}$. The posets $\left(S_{n}, \leq_{R}\right)$ and $\mathcal{P}\left(\mathcal{G}^{A}\right)$ are isomorphic.

Proof: It is enough to show that $I S(\mathcal{G})=\left\{\operatorname{Inv}(\sigma) \mid \sigma \in S_{n}\right\}$. The inclusion " $\supseteq$ " is based on a verification using Proposition 3.1.1

To give a sketch of the proof of the reverse inclusion, we recall a classical concept about permutations. Set $\sigma \in S_{n}$ and $1 \leq a<b \leq n$ two integers. We say that $a$ and $b$ are adjacent in $\sigma$ if and only if $\sigma^{-1}(a)+1=\sigma^{-1}(b)$. This notion is linked to the weak order thanks to the following property: we have that $\sigma \leq_{R} \omega$ with $\ell(\omega)=\ell(\sigma)+1$ if and only if $\omega$ can be obtained from $\sigma$ by exchanging the position of two adjacent entries of $\sigma$. In that case, we say that $\omega$ covers $\sigma$ and we denote it $\sigma \triangleleft \omega$. This can be transposed in the context of the inversion sets as follows: we have that $\sigma \triangleleft \omega$ if and only if $\operatorname{Inv}(\omega)=\operatorname{Inv}(\sigma) \cup\{(a, b)\}$ where $a$ and $b$ are two adjacent entries of $\sigma$.

Now consider $B \in I S\left(\mathcal{G}^{A}\right)$ and $L=\left[\left(a_{1}, b_{1}\right), \ldots,\left(a_{N}, b_{N}\right)\right] \in P S\left(\mathcal{G}^{A}\right)$ such that $B=\left\{\left(a_{1}, b_{1}\right), \ldots\right.$, $\left.\left(a_{k}, b_{k}\right)\right\}$. Set $B_{0}=\emptyset$, and $B_{i}=\left\{\left(a_{1}, b_{1}\right), \ldots,\left(a_{i}, b_{i}\right)\right\}$ for all $1 \leq i \leq k$. We can show recursively that $B_{i}$ is the inversion set of a permutation. 
- If we denote $I d=[1,2, \ldots, n]$, then $B_{0}=\operatorname{Inv}(I d)$.

- By definition of $\mathcal{G}^{A}$, we have that $\left(a_{1}, b_{1}\right)$ is of the form $(j, j+1)$ for some $j \in\{1, \ldots, n-1\}$. Since $j$ and $j+1$ are adjacent in $I d$, we have that $I d \triangleleft \sigma_{1}:=[1, \ldots, j+1, j, \ldots, n]$, thus $\operatorname{Inv}\left(\sigma_{1}\right)=\{(j, j+1)\}=\left\{\left(a_{1}, b_{1}\right)\right\}=B_{1}$.

- Now, consider $\mathcal{G}_{2}^{A}$ obtained with the peeling process after we picked $\left(a_{1}, b_{1}\right)$. Since $L \in P S\left(\mathcal{G}^{A}\right)$, $\left(a_{2}, b_{2}\right)$ is erasable in $\mathcal{G}_{2}^{A}$. Then we can show that $a_{2}$ and $b_{2}$ are adjacent in $\sigma_{1}$, hence $\sigma_{1} \triangleleft \sigma_{2}$, where $\sigma_{2}$ is obtained from $\sigma_{1}$ by exchanging the positions of $a_{2}$ and $b_{2}$. Hence $\operatorname{Inv}\left(\sigma_{2}\right)=\operatorname{Inv}\left(\sigma_{1}\right) \cup$ $\left\{\left(a_{2}, b_{2}\right)\right\}=\left\{\left(a_{1}, b_{1}\right),\left(a_{2}, b_{2}\right)\right\}=B_{2}$.

- The sequel of the induction is similar: if there is $\sigma_{j}$ such that $\operatorname{Inv}\left(\sigma_{j}\right)=\left\{\left(a_{1}, b_{1}\right), \ldots,\left(a_{j}, b_{j}\right)\right\}$, then we show that $a_{j+1}$ and $b_{j+1}$ are adjacent in $\sigma_{j}$, and we construct $\sigma_{j+1}$ as before. The exact proof is quite technical and is essentially based on refinements of the arguments introduced by Edelman and Greene about balanced tableaux of staircase shape. The interested reader can look at [9] for a proof using another terminology.

As an immediate consequence, $B=B_{k}=\operatorname{Inv}\left(\sigma_{k}\right)$. This concludes the proof.

\subsection{A combinatorial model for reduced decompositions in $A_{n-1}$}

Remark 4.2.1 For a more detailed description of the content of this section, the reader can look at [9].

This construction provides a new combinatorial interpretation of the elements of $\mathcal{R}(\sigma)$ for each $\sigma \in S_{n}$. That is, consider $\sigma \in S_{n}$ and $B \in I S\left(\mathcal{G}^{A}\right)$ such that $B=\operatorname{Inv}(\sigma)$. We may associate to $B$ a canonical digraph structure and an OCV by "restriction" of the structure of $\mathcal{G}^{A}$. That is, the sub-diagram $B$ inherit of the digraph structure of the original diagram. Moreover, since $B$ is an initial section, the restriction of $\theta$ to this sub-diagram is an OCV of it. The next figure depict this restriction on the example of $[4,1,3,5,2]$ (the integers in the boxes represent the values of $\theta$ on each box).

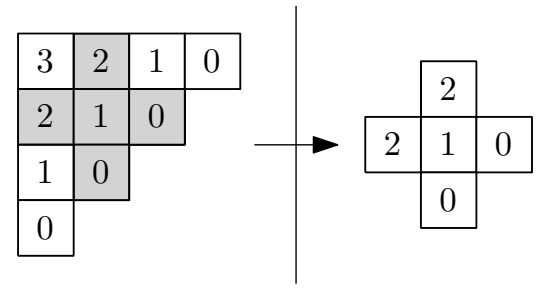

We will denote $P S(B)$ the set of all the peeling sequences of the sub-graph and OCV induced by $B$.

Proposition 4.2.1 Set $\sigma \in S_{n}$ and $B$ its associated diagram. There is a bijection between $\mathcal{R}(\sigma)$ and $P S(B)$.

Proof: Recall that a maximal chain from $I d$ to $\sigma$ (in the weak order) is a sequence $\left(\sigma_{j}\right)_{1 \leq j \leq k}$ such that $I d \triangleleft \sigma_{1} \triangleleft \ldots \triangleleft \sigma_{k}=\sigma$. There is a canonical bijection between elements of $\mathcal{R}(\sigma)$ and maximal chains from $I d$ to $\sigma$. That is, set $s_{i_{1}} \cdots s_{i_{k}} \in \mathcal{R}(\sigma)$ and denote $\sigma_{j}=s_{i_{1}} \cdots s_{i_{j}}$, then we have $I d \triangleleft \sigma_{1} \triangleleft \ldots \triangleleft \sigma_{k}=\sigma$. Conversely, if $\sigma \triangleleft \omega$, we have that there exists $s_{p}$ such that $\omega=\sigma s_{p}$ by definition of the weak order, thus a maximal chain leads to a reduced decomposition of $\mathcal{R}(\sigma)$. 
Now set $L=\left[\left(a_{1}, b_{1}\right), \ldots,\left(a_{k}, b_{k}\right)\right] \in P S(B)$, and denote $B_{i}=\left\{\left(a_{1}, b_{1}\right), \ldots,\left(a_{i}, b_{i}\right)\right\}$. By construction of the isomorphism between $\left(S_{n}, \leq_{R}\right)$ and $\mathcal{P}\left(\mathcal{G}^{A}\right)$, there exists $\sigma_{i}$ such that $\operatorname{Inv}\left(\sigma_{i}\right)=B_{i}$. Hence we have $\emptyset \subset \operatorname{Inv}\left(\sigma_{1}\right) \subset \ldots \subset \operatorname{Inv}\left(\sigma_{k}\right)$. Then we transpose this expression to the weak order, and it becomes $I d \triangleleft \sigma_{1} \triangleleft \ldots \triangleleft \sigma_{k}=\sigma$. To summarize, we associated a maximal chain (from $I d$ to $\sigma$ ) to each element of $P S(B)$, and we can show that it is a bijection. This concludes the proof.

Example 4.2.1 We conclude by an example of application of this bijection to $\sigma=[4,1,3,5,2]$. Denote $B$ the diagram associated with $\sigma$, and consider $L=[(2,3),(2,4),(3,4),(1,4),(2,5)] \in P S(B)$. The maximal chain associated with $L$ is: $\sigma_{1}=[1,3,2,4,5]=s_{2}, \sigma_{2}=[1,3,4,2,5]=\sigma_{1} s_{3}, \sigma_{3}=$ $[1,4,3,2,5]=\sigma_{2} s_{2}, \sigma_{4}=[4,1,3,2,5]=\sigma_{3} s_{1}$, and $\sigma_{5}=[4,1,3,5,2]=\sigma_{4} s_{4}$. Thus the associated reduced decomposition is $s_{2} s_{3} s_{2} s_{1} s_{4}$. The next figure describes completely the bijection in the case of $[4,1,3,5,2]$ (for the reader convenience, peeling sequences are represented by tableaux).

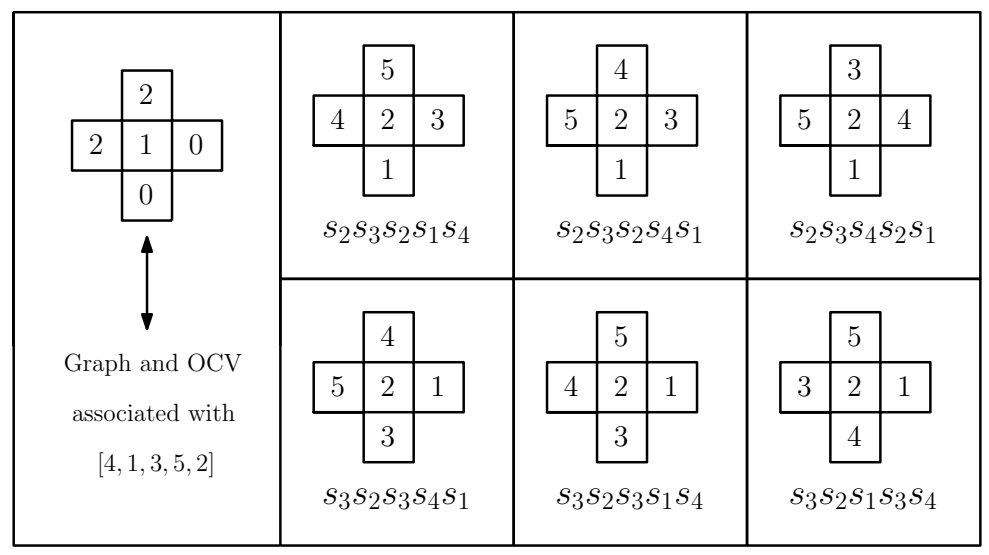

\subsection{Application to the Stanley series of type $A$}

The graphical interpretation of the reduced decomposition of a permutation as set of tableaux leads to a natural question: can we define a good generalization of the concept of semi-standard Young tableaux in this context? If this is the case, what does the associate formal power series look like ?

A natural starting point is to consider tableaux defines by a "column-strictness" condition as in [5].

Definition 4.2 Set $\sigma \in S_{n}$ and $B$ its associated diagram. A tableau $T$ of shape $B$ is a sequence of integers indexed by the elements of $B$, that is $T=\left(t_{c}\right)_{c \in B}$. We say that $T$ is $\sigma$-semi-standard if and only if there exists $L=\left[c_{1}, \ldots, c_{k}\right] \in P S(B)$ such that:

1. for all $i<j, t_{c_{i}} \leq t_{c_{j}}$;

2. for all $i<j$ such that $c_{i}$ and $c_{j}$ lies in the same column of $B, t_{c_{i}}<t_{c_{j}}$.

The set of the $\sigma$-semi-standard tableaux is denoted $S S T(\sigma)$. Finally, we denote $x^{T}$ the monomial $\prod_{c \in B} x_{t_{c}}$.

It appears that the tableaux in $S S T(\sigma)$ and the column-strict balanced labellings of the Rothe diagram of $\sigma$ introduced in [5], are basically the same objects. That is, one can travel from one point of view to another just by swapping some lines of the tableaux, as depicted below. 


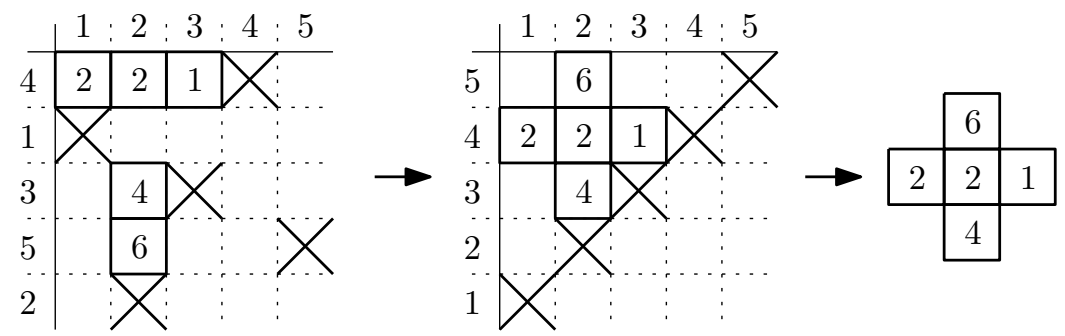

Hence, as an immediate consequence of (Theorem 4.3, [5]), we have the following Theorem.

Theorem 4.3 For all $\sigma \in S_{n}, F_{\sigma}\left(x_{1}, x_{2}, \ldots\right)=\sum_{T \in S S T(\sigma)} x^{T}$, where $F_{\sigma}$ is the Stanley series of type A associated with $\sigma$ and defined by

$$
F_{\sigma}\left(x_{1}, x_{2}, \ldots\right)=\sum_{s_{i_{1}} \cdots s_{i_{\ell}(\sigma)} \in \mathcal{R}(\sigma)} \sum_{\substack{b_{1} \leq b_{2} \leq \ldots \leq b_{\ell(\sigma)} \\ b_{j}<b_{j+1} \text { if } \\ i_{j}>i_{j+1}}} x_{b_{1}} x_{b_{2}} \cdots x_{b_{\ell(\sigma)}} .
$$

\section{Other cases}

In the previous section we studied the case $A$ in details, and showed how it naturally leads to a combinatorial interpretation of the associated Stanley series. A similar process can be performed in the $\widetilde{A}$ case, and leads to an analogous combinatorial interpretation of the reduced decompositions in $\widetilde{A}$, and of the corresponding Stanley series (using [10]) introduced by Lam in [6]. In that case, the underlying graph can be seen as an infinite version of $\mathcal{G}^{A}$, which is rolled around a cylinder. For a graphical representation, see the following figure.

In the case of the weak order on the type $B$, the digraph is a bit more complicated and can be visualize as a shifted diagram, where the digraph structure is given by the shifted hooks. In that case, the study of the associated formal power series which arise is undone, and the author will focus on that topic later. To conclude, we mention that some clues suggest that a description of the weak order on any Coxeter group with this theory is possible, and this will be the subject of a later publication.

We finish by giving, without justification, the graphical representation of some of these objects.

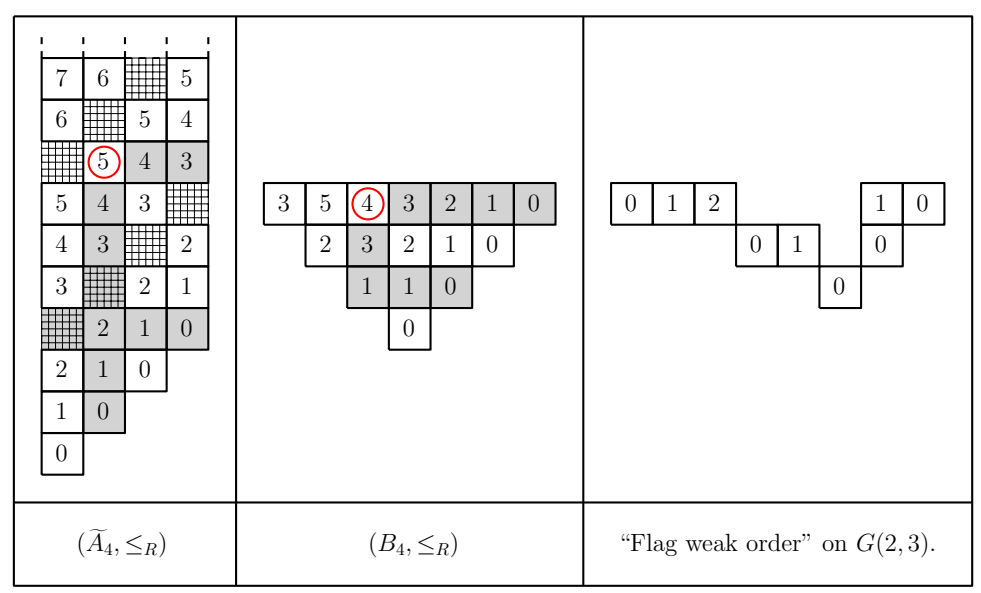




\section{References}

[1] R. Adin, F. Brenti, Y. Roichman, Flag weak order on wreath products. Sém. Lothar. Combin. 67 (2011/12), B67e, 20pp.

[2] A. Bjorner, Orderings of Coxeter groups. Contemp. Math., 34, Amer. Math. Soc., Providence, RI, 1984.

[3] A. Bjorner, F. Brenti, Combinatorics of Coxeter groups. Springer, New York, NY, 2005.

[4] P. Edelman, C. Greene, Balanced tableaux. Adv. Math., 63, 42-99, 1987.

[5] S. Fomin, C. Greene, V. Reiner, M. Shimozono, Balanced Labellings and Schubert Polynomials. Europ. J. Combin., 18, 373-389, 1995.

[6] T. Lam, Affine Stanley symmetric functions. Amer. J. Math., 128(6):1553-1586, 2006.

[7] R. P. Stanley, On the number of reduced decompositions of elements of Coxeter groups. Europ. J. Combin., 5, 359-372, 1984.

[8] R. P. Stanley, Enumerative combinatorics. Vol. 1, volume 62 of Cambridge studies in advanced mathematics. Cambridge university press, Cambridge, 1999. With a foreword by Gian-Carlo Rota and appendix 1 by Sergey Fomin.

[9] F. Viard, A natural generalisation of balanced tableaux. Preprint arXiv.org:1407.6217, 2014 (24 pages).

[10] H. Yoo, H. Yun, Balanced labellings of affine permutations. DMTCS Proceedings, north America, FPSAC 2013. Available at: www.dmtcs.org/dmtcsojs/index.php/proceedings/article/view/dmAS0166. 